\title{
From Index to Network: Topic Maps in the Enhanced Networked Monographs Project [submitted version]
}

Alexandra Provo

Final version: Provo, A. (2019). From index to network: topic maps in the Enhanced Networked Monographs project. The Indexer: The International Journal of Indexing, 37(1), 13-35. https://doi.org/10.3828/indexer.2019.3

\section{Abstract}

The Enhanced Networked Monographs (ENM) project was an experimental publishing project funded by The Andrew W. Mellon Foundation and carried out from 2015-2018. Over the course of the project, we developed methods to extract topics from indexes, built tools to curate the result of the integration, and created a platform for reading. This article will discuss the creation of the ENM topic map, a meta-index created by combining many individual back-of-book indexes using the Topic Curation Toolkit. 


\section{Introduction}

The Enhanced Networked Monographs (ENM) project was an experimental publishing project funded by The Andrew W. Mellon Foundation and carried out from 2015-2018. A collaboration between NYU Press and Digital Library Technology Services at NYU's Division of Libraries, ENM's purpose was to provide free, web-based access to 110 back-list scholarly monographs representing various disciplines and domains of knowledge, drawn from NYU Press, the University of Minnesota Press, and the University of Michigan Press. Over the course of the project, we developed methods to extract topics from indexes, built tools to curate the result of the integration, and created a platform for reading. The tool for extraction and curation, called the Topic Curation Toolkit, was developed by Infoloom, and is based on the Topic Maps architecture. This article will discuss the creation of the ENM topic map, a meta-index created by combining many individual back-of-book indexes using the Topic Curation Toolkit. ${ }^{1}$

\section{Brief overview of topic maps}

Originally developed in the 1990s to address the need for standardized terminology and master indexes for UNIX documentation and printed manuals, Topic Maps is an ISO standard (ISO 13250) for representing data about concepts. In 2000, the model was also expressed in XML (Newcomb, 2003). Though the standard was originally born out of interest in back-of-book index structures, the developers soon realized that it could apply to many other types of controlled vocabulary such as thesauri and taxonomies. ${ }^{2}$

The ENM project did not adhere exactly to the specifications in the ISO standard, nor did it use the XML expression of Topic Maps. Rather, the underlying data model of Topic Maps guides the structure of the subject database in ENM. ${ }^{3}$ Conceptually, a topic map is an interconnected dataset that sits above or on top of a source, facilitating navigation between parts of the source. A source could be a single document consisting of multiple parts (such as a book and its pages), or a collection of multiple documents.

\footnotetext{
${ }^{1}$ Many thanks to Michel Biezunski for reviewing this article and collaborating on this project, and to NYU's ENM team members for all of their hard work on the ENM topic map and prototypes: David Arjanik, Laura Henze, Monica McCormick, Carol Kassel, and David Millman.

${ }^{2}$ For a fuller explanation and consideration of the history of topic maps, see the article by Michel Biezunski in issue $\mathrm{X}$ of The Indexer.

${ }^{3}$ In this article, I will use capital letters to refer to the ISO standards and lowercase to refer to the ENM implementation, to distinguish the formal specification from projects like ours that have a general topic map shape but do not use the standard syntax or all elements of the Topic Maps model.
} 
A topic map is comprised of topics and relations between topics or between topics and sources. The latter form of association is called an occurrence. Topics themselves are comprised of names and can have types. In book index terms, a topic is a heading, an association relation is a "see" or "see also" cross-reference, and occurrences are the page number locators listed for each heading. Topic map elements can also be scoped; as with many features of topic maps, scope is flexible and the nature of its use is left to implementers. In the ENM project, we elected to use scopes as qualifiers, which in an index one would encounter as parentheticals in index headings.

\section{Related work}

ENM is not the first project to provide an integrated index based on back-of-book indexes, although it is one of just a few. A key example is a research project out of the University of British Columbia called the Indexer's Legacy project, active in the early 2010 s. This project took PDFs from specific knowledge domains as its source content. Indexer's Legacy sought to investigate the potential added-value to search of the domain knowledge found in back-of-book indexes (Huggett and Rasmussen, 2011a). Curated collections of digital texts were parsed by specialized scripts and software, called the Meta-Dex suite (Huggett and Rasmussen, 2011b). One of the results of the project was a prototype user interface, which provided search, index entry pages, views of book page content, and domain summaries, including calculations such as shared entries and "coherence," or "the degree to which the books of the domain contain the same entries" (Huggett and Rasmussen, 2012).

Another example is the American Society of Indexing's Publications Mashup (ASI, 2013). This is an HTML site providing a hyper-linked alphabetical list of topic terms, as well as an index view that presents headings, subheadings, book, and locator information. For this project, existing eBook indexes were copied using Cindex software, where book title abbreviations were added to the locators. Then, the individual indexes were merged into a master index file, which was then curated by hand by a professional indexer (Ream and Wyman, 2014).

\section{Topic curation workflows and the Topic Curation Toolkit}

\section{Topic curation toolkit and topic record structure}

The ENM topic map was generated using the Topic Curation Toolkit (TCT). Developed by Infoloom, the TCT is an open source software application built on the Django web framework with a Postgres relational database. The front end was built using Google's AngularJS, version 
1. Code has been made available under an open source Apache 2.0 license on the NYU Libraries Github page.

The sources for the ENM topic map are indexes from long-form scholarly monographs published by University Presses. Texts were chosen from three University Presses (NYU Press, University of Michigan Press, and University of Minnesota Press) on the basis of whether or not the text was already open access, or whether the Press wanted to make the title available open access. These three presses are all engaged in projects related to digital monograph publishing, funded by the Andrew W. Mellon Foundation. Unlike the Indexer's Legacy project or the ASI publications mashup, ENM made no attempt to select texts from the same subject domain. Domains include the academic fields of literary analysis, philosophy, law, media studies, and gender studies, among others. The texts were published between 1987 and 2016, with the largest number published in 1998. Unlike the Indexer's Legacy project, ENM takes EPUB files as its source content. Of the 113 files submitted for inclusion, 104 titles are included in the ENM corpus - the remainder did not have page number indicators in the EPUB version, and therefore could not be added to the topic map, which uses page numbers as locations for topics. Of the 104 included titles, only 89 titles contributed to the topic map itself, since the remainder did not have indexes, which are required in order to generate topics.

The TCT software creates topic records by parsing back-of-book EPUB indexes and generating a record from each index heading. The parser also processes the EPUB page text and saves each page as unformatted text content tied to a "location" in the database. As in the Meta-Dex suite, index subentries were "expanded" in ENM by concatenating the main part of the entry with the subentry. For example, in the index for the book Can Unions Survive? the heading Concerted activity has two subentries: constructive and individual conduct (figure 1). In the TCT, the names for those subentries are concatenated and separated by a double dash, a display style common in library subject headings, becoming Concerted activity -- constructive and Concerted activity -- individual contact.

\author{
Compensatory relief against employer, 153 \\ Computers, 40, 41, 84 \\ Concerted activity, 136 \\ constructive, 147; \\ individual conduct, 130 \\ Conglomerates, 46 \\ Congress of Industrial Organizations \\ (CIO), 28, 31 \\ Consumer activity: boycotts, 11, 14, 93 \\ handbilling, 146
}

Figure 1. Index example from Can Unions Survive? 
To create the linkages between topics, which is what gives a topic map its network structure, the TCT makes use of algorithms to relate topics to one another. Python scripts in TCT take the individual words in each topic name and strip them of suffixes and some punctuation in order to turn them into essentialized "tokens." The automated, rule-based algorithms for relating topics are outlined below.

\begin{tabular}{|c|c|}
\hline Relation rule & Description \\
\hline Containment & $\begin{array}{l}\text { Ignores stopwords defined in the system. If all } \\
\text { of one name string's tokens are contained in } \\
\text { the name string of another topic, a relation is } \\
\text { made. For example, the name string Ambition } \\
\text { - narcissism contains Narcissism. }\end{array}$ \\
\hline Multiple tokens & $\begin{array}{l}\text { Automatically generated by the TCT } \\
\text { algorithm; ignores stopwords. The general } \\
\text { rule is that if two topics have three or more } \\
\text { tokens in common, they are related; for } \\
\text { example, the topic abolitionism -- and } \\
\text { American radical tradition has three tokens in } \\
\text { common with gay liberation -- and American } \\
\text { radical tradition. The tokens in common are } \\
\text { American, radical, and tradition. } \\
\text { If a topic is a subentry, the multiple tokens rule } \\
\text { is slightly different. For subentries to be } \\
\text { related to one another, they must have at } \\
\text { least one token in the subentry portion of the } \\
\text { name string in common. For example, } \\
\text { Vietnam Veterans Memorial -- } 1988 \text { Veterans } \\
\text { Day ceremony is related to Vietnam Veterans } \\
\text { Memorial -- dedication ceremonies because } \\
\text { these two subentries share the token } \\
\text { ceremony. }\end{array}$ \\
\hline Main- and sub-entry & $\begin{array}{l}\text { The subentry relation is automatically } \\
\text { generated by the TCT, but it is based on } \\
\text { something the human indexer has done. In an } \\
\text { index, a subentry is usually separated from a } \\
\text { main entry by punctuation or an indent. In an } \\
\text { example abolitionism -- and American radical } \\
\text { tradition, abolitionism is the main entry and }\end{array}$ \\
\hline
\end{tabular}




\begin{tabular}{|c|c|}
\hline & $\begin{array}{l}\text { and American radical tradition is the } \\
\text { subentry. It looks like this in a paper index: } \\
\text { abolitionism } 23,27,56 \\
\text { and American radical tradition } 38,44,78 \\
\text { and the South } 38,89 \\
\text { The TCT "expands" subentries, meaning it } \\
\text { understands that the indent indicates a } \\
\text { subentry, so it takes the implied main entry } \\
\text { abolitionism and puts it in the name string, } \\
\text { resulting in abolitionism - and American } \\
\text { radical tradition. }\end{array}$ \\
\hline See & $\begin{array}{l}\text { A see relation is a human indexer-created } \\
\text { cross-reference. It is sometimes used to } \\
\text { indicate a synonym, but not always. If the TCT } \\
\text { detects the word "see" in an EPUB index, it } \\
\text { generates a see relation to the name string } \\
\text { that succeeds the word "see". For example, } \\
\text { the entry Hebrew Book of Enoch. See Sefer } \\
\text { Hekhalot creates a relation between Hebrew } \\
\text { Book of Enoch and Sefer Hekhalot. }\end{array}$ \\
\hline See also & $\begin{array}{l}\text { A see also relation is a human indexer-created } \\
\text { cross-reference. If the TCT detects the words } \\
\text { "see also" in an EPUB index, it generates a } \\
\text { see relation to the name string that succeeds } \\
\text { the words "see also". For example, conjure } \\
\text { cosmology see also African diasporic } \\
\text { cosmologies. }\end{array}$ \\
\hline
\end{tabular}

The elements that may be included in a topic record are outlined below. Starred $\left(^{*}\right)$ elements are automatically populated by the TCT. These same elements can be seen in the TCT editorial interface in figure 2.

- $\quad$ *Internal topic ID

- Topic name

- *Topic name string

- Topic name scope (qualifier)

- Topic display, or preferred, name 
- Topic type

- *Topic name string

- Topic name scope (qualifier)

- *Topic relationships with other topics

- ${ }^{*}$ Topic occurrences (links to the print page number where the topic is mentioned in a given book)

- Topic weblinks

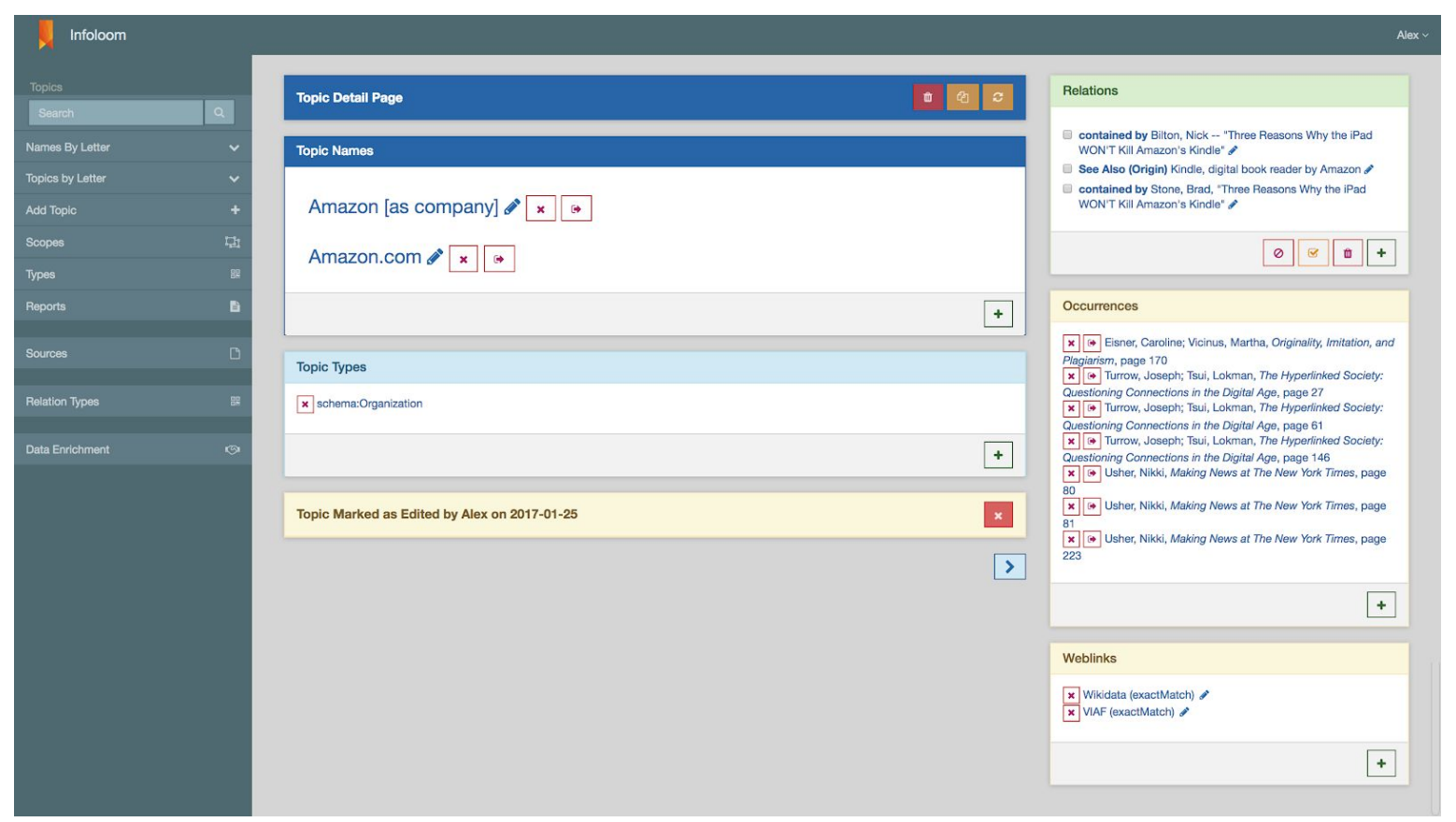

Figure 2. Screenshot of a topic record for Amazon.com in the TCT editorial interface.

As part of the creation of an ENM topic map Metadata Application Profile (MAP), an object-role model (ORM) diagram was created to convey topic map elements, relations, and whether elements are required or mutually exclusive in the system (see figure 3 ). The main constraints of the data model are that topics must have at least one name, that names must consist of a string and a scope element, and that no two topic records may have the same name string and scope combination. 


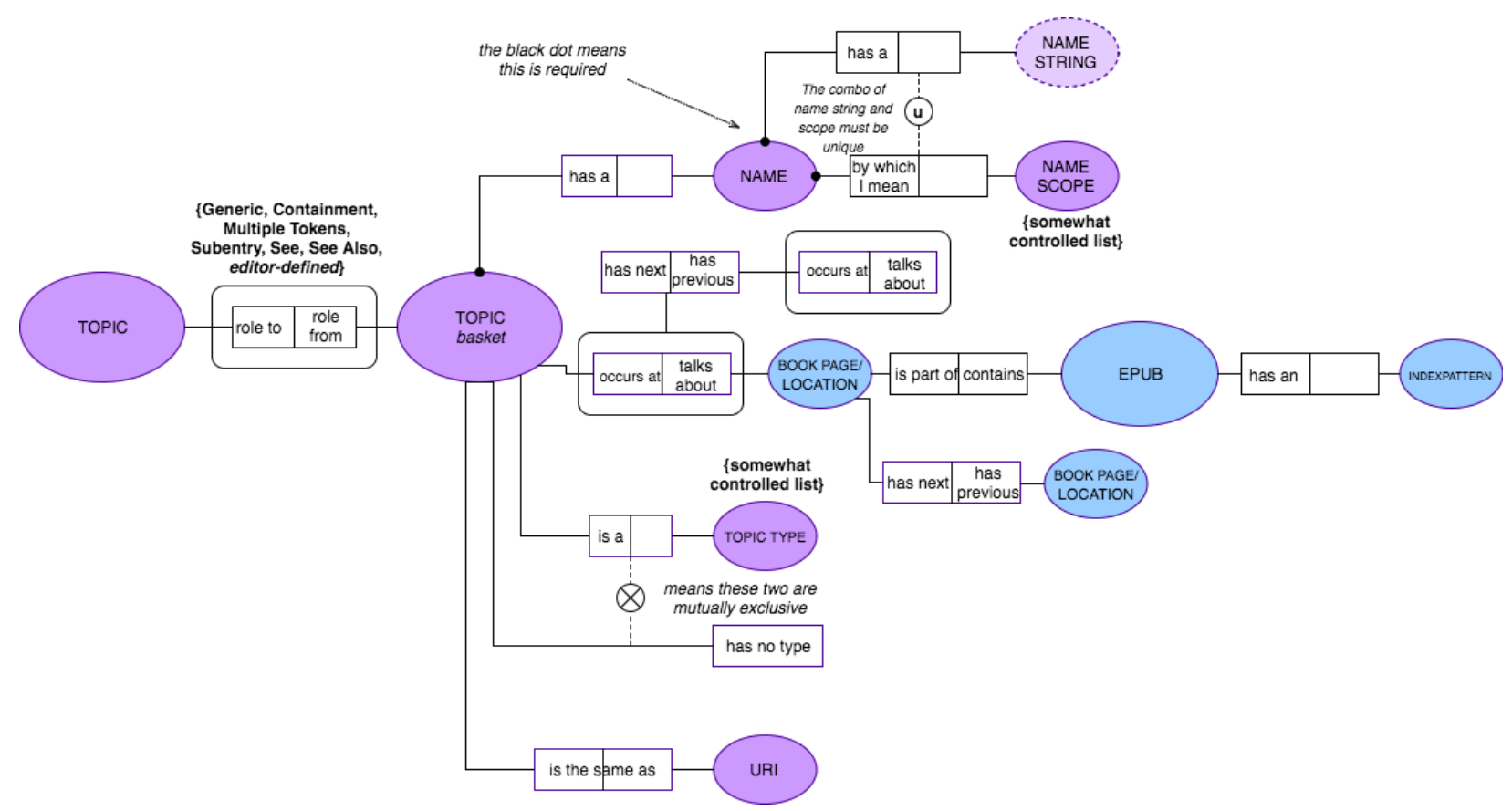

Figure 3. Topic map record modeled in ORM.

\section{Topic curation workflows}

The workflow for creating and managing the ENM topic map is a mix of machine processing and human curation. The TCT, as outlined above, merges and links topics, and a human editor then reviews and performs quality control. As we embarked on this aspect of the project, we wanted to understand what it would it take to make a topic map that works. Does the TCT process topics well enough so that we can more or less leave it alone, and if not, how much human intervention is needed?

The TCT editorial interface includes views showing alphabetical lists of topics as well as all topic names, individual topic records, and book content broken up by page (called an occurrence). The alphabetical list views include options for sorting alphabetically, by number of relations, and by number of occurrences. In the individual topic record views, arrow buttons provide navigation between topics. A pencil icon next to many of the fields indicates that it can be directly edited. There are also buttons to add new data, a "re-run relations" button, and two review buttons that indicate whether a topic is unreviewed, reviewed, or edited. In the occurrence (or book content) view, arrow buttons provide sequential navigation between the pages of the book, and topic arrow buttons provide non-sequential navigation between occurrences of that topic on various pages in the given book as well as other books containing that topic.

The first workflow created was a TCT workflow for reviewing and editing topics alphabetically. As the ENM project's Digital Production Editor, I selected a letter in the TCT, sorted topics alphabetically within that letter, and proceeded down the list by opening the first topic record and then navigating to the next one using arrow buttons within the topic record. Between 
January and June of 2017 , I checked about 15,800 topics, or a little over one third of the topic map. 12,647 topics were marked as reviewed but unchanged, while 3,151 were edited.

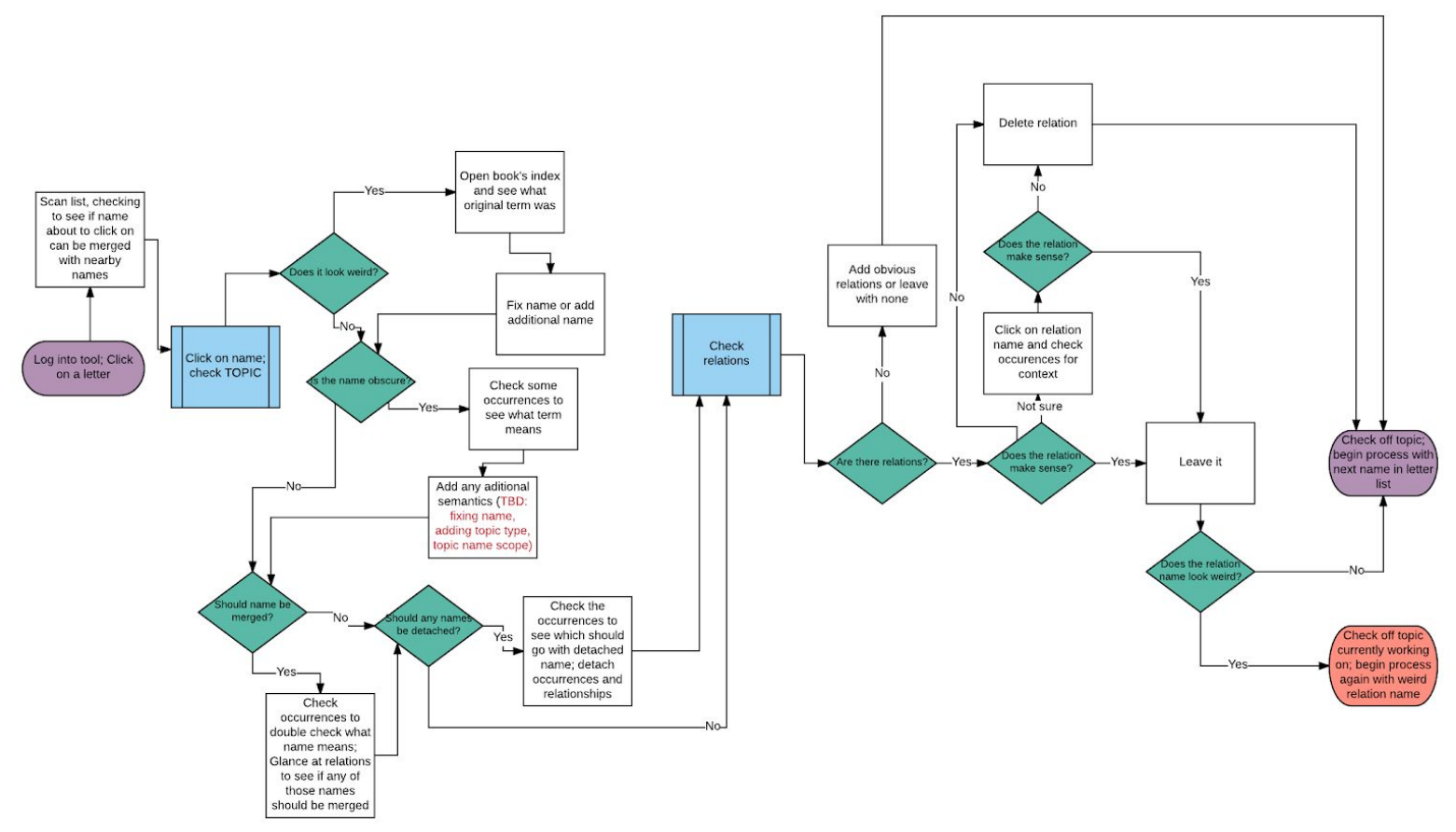

Figure 4. Alphabetical workflow diagram.

One of the outcomes of this alphabetical workflow test was that it helped the team discover issues and make recommendations for improving the TCT. For example, we found that if a main entry did not list any locators, sometimes subentries parsed incorrectly. This was a problem we were able to fix in the TCT software, as its index parser operates using index patterns which are configurable. In contrast, another issue arose if page numbers were linked in unexpected ways. This is an issue that would be best resolved in the EPUB markup itself. Likewise, some EPUBs we worked with had indentation and line break issues, which resulted in strange or incorrectly aggregated topics.

Other, more semantic problems arose as unintended consequences of rules that otherwise functioned correctly. When the TCT parses indexes, it generates relations or links between topics based on shared tokens (essentially, words) in topic names. The rule is that a link is formed if three or more tokens are shared. For a set of Zora Neale Hurston topics, this created many relationships between subentries, which we determined to be noise, since the user would be able to navigate between subentries by visiting the page of the main entry (or parent topic) which serves as a hub. To address this issue, we modified the rule so that subentries with the same main entry did not relate if the three words were part of the main entry portion of the topic name. Another issue under the category of "rules gone awry" is that the TCT's containment rule 
for making relations resulted in Biblical names being related to many personal names. As well, abbreviations caused ambiguity and either caused irrelevant relations to be created, or the collapse of occurrences about two different people, as can be seen in a screenshot of a topic that combines locators for Stephen Hall and Stuart Hall.

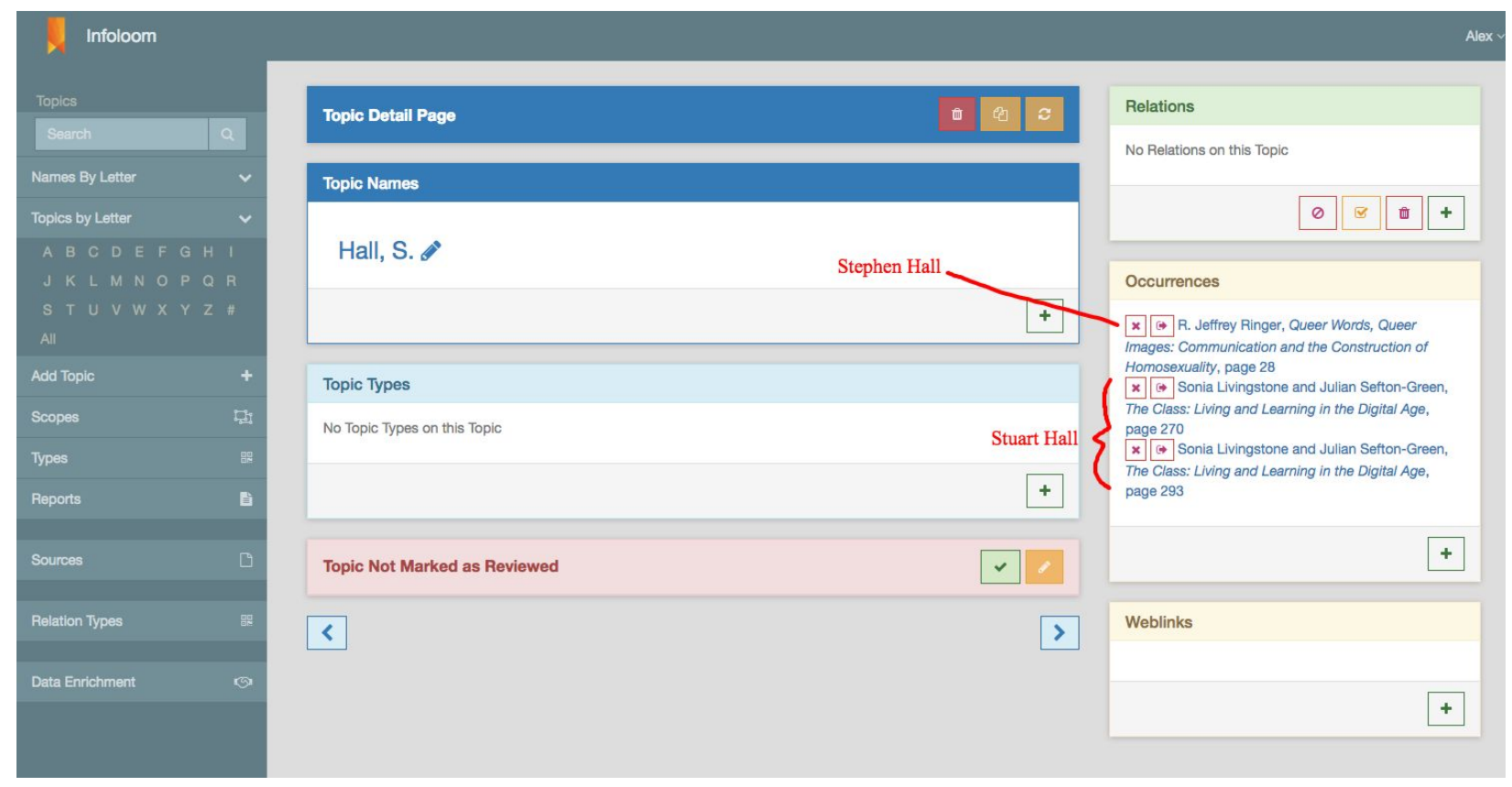

Figure 5. Screenshot of record conflating Stephen Hall and Stuart Hall.

Implied context emerged as a problem for concepts as well as people. For example, in the book Please Don't Wish Me a Merry Christmas, the index entry Civil War points the reader to the more specific topics American Civil War and English Civil War via a "see" cross-reference. The occurrences from other books on the Civil War topic page are about the American Civil War. The term Civil War takes on different meanings in these different book contexts. Homographs also emerged as a thorny issue, with meaning becoming collapsed in the same topic. In the above examples, the change in context from a small scale (one book) to a larger scale (a corpus) causes semantic problems. 


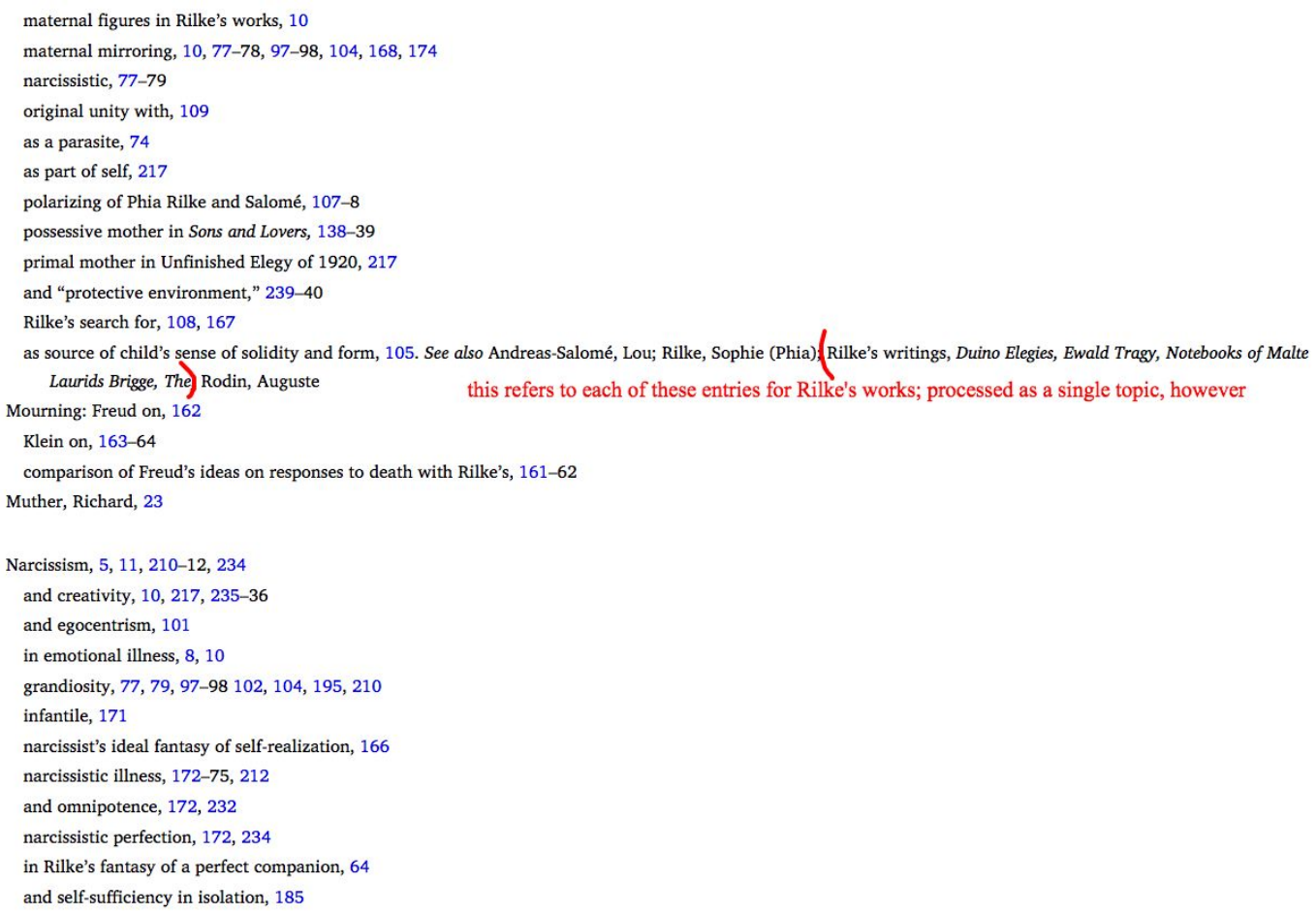

Figure 6. Rilke's writings issue.

Other semantic issues arise from the switch in context from a human reader to a machine reader. For example, in the entry for Michigan Department of Corrections in the book Is William Martinez Not our Brother?, a "see also" cross-reference points the reader to specific names of institutions and provides an example; however, the TCT reads "specific names of institutions" as its own topic name. Likewise, in the index entry for Mother -- as source of child's sense of solidity and form in the book The Beginning of Terror, a see also cross-reference lists Rilke's writings but in a format that breaks from the index pattern the parser was expecting (figure 6), resulting in the tool recognizing the list as one topic name. Under the heading Arrogant-vindictive (aggressive) solution from the book Imagined Human Beings (figure 7), while a human reader could easily understand where to navigate in the index to find the analyses listed in the "see" cross-reference provide, the TCT does not have the ability to understand this nuance and instead looks for an exact string match. For this reason as well, the TCT created a new topic for the characters listed, since elsewhere in the index, characters such as Pip are referred to with a parenthetical qualifier "Great Expectations." The result was that two separate topics were created for this same concept.

\footnotetext{
Antony (Antony and Cleopatra), 22, 93

Antony and Cleopatra (Shakespeare), 22, 93, 212

Arrogant-vindictive (aggressive) solution: defensive strategies of, 22-24, 253-54; examples of in literature, 24. See analyses of Catherine Earnshaw; Creon; Hedda Gabler; Michael Henchard; Pip; Shylock Auerbach, Erich, 196

Austen, Jane, 5, 13, 22, 27, 29, 264, 271 n. 1; Heading is Pip (Great Expectations) criticism of, 269
}

Figure 7. Arrogant-vindictive (aggressive) solution issue. 
Another issue that is easily parsed by a human reader but presents problems for machines is the convention of creating topics by combining two almost equivalent words. As in other examples, the change in context from a small scale (one book) to a larger scale (a corpus) causes semantic problems. Combination topics consist of distinct but related concepts, or different parts of speech. These are frequently separated by a slash, but may also be indicated in other ways. For example, indexers have created topics like advertising/advertisements; Vietnam/Vietnamese; anarchism/anarchists; democracy/democratization; Narcissism/narcissistic. An example of two distinct concepts being combined is Race/ethnicity. Two non-slash examples found in the ENM corpus are Behavior(al) and Arab, Arabic. While it might seem reasonable to combine these terms and merge topics, an index in one book may have two separate entries with different locators for terms that have been combined in another book. For example, there is a democracy/democratization topic in the book Framed: The New Woman Criminal in British Culture at the Fin de Siecle, but in the book Internationalizing "International Communication" democracy and democratization are separate topics with different locators. Somewhat relatedly, flipping or double posting also created issues: within the same index, sometimes topics that contained the same words in a different order had slightly different locators. In some cases, these appear to be the same concept, but other cases were not so clear.

The errors and issues described above were collected and used to draft an editorial policies document. Modeled on the structure of Agile Modeling (Ambler, 2002), the policies document outlines values, principles and practices. The values identified were:

- Automation over human curation

- Serendipity over rigidity

- Transparency and mitigation of bias

- Autonomy of the user (potential conflict with Serendipity over rigidity)

- Accuracy of content (potential conflict with Automation over human curation)

The last three of these values are derived from scholarship on Value Sensitive design in information systems (Friedman, Kahn, and Borning, 2006).

Principles identified were more specific than values, and include:

1. Pay attention to the impact of editorial intervention on the end-user

2. Minimal involvement of the editor

3. Privilege serendipity through loose semantics

4. Minimize misleading or irrelevant relations between topics

5. Work to get through all topics

6. Acknowledgement of non-neutrality

7. Proceed as if we are going to continue adding terms and indexes to the topic map dataset. 


\section{More involved editing in special cases}

The policies are the practices followed by the Digital Production Editor while editing topics. For example, one policy under the category of splitting topics has to do with splitting proper nouns and general concepts. This came out of the homograph issue described earlier. The example given under this policy is the topic Nation. Some occurrences refer to the concept of a nation, while others referred to the publication The Nation. This collapse happened because the TCT ignores stopwords like the, a, etc. Taking into account the project's values of autonomy and accuracy, we imagined that a user trying to read about The Nation would become frustrated with having to sift through occurrences about the general concept.

\section{Targeted curation}

The patterns and issues that informed the values, principles, and practices outlined above were used to determine different criteria by which to slice the topic map into smaller subsets. As mentioned previously, only about 3,000 topics out of about 15,800 were edited, so the team was interested to see if it would be possible to more precisely target topics that might require detailed intervention. For example, the realization that abbreviated names caused semantic problems was the impetus for creating an abbreviated names workflow. In this workflow, a Python script that uses regular expressions to identify abbreviated names following the pattern [LAST NAME], [INITIALS]. This list was then used to generate links to individual TCT records that were then edited manually.

In part as an alternative to the earlier A-Z workflow, the Digital Production Editor also determined a subset of topics that could be considered "important." Importance was considered in terms of participation in the topic map graph network, based on a linkage metric described in Xavier Ochoa and Erik Duval's 2009 article "Automatic evaluation of metadata quality in digital repositories" (Ochoa and Duval, 2009), which is listed in the DLF Metadata Assessment Working Group's Environmental Scan (DLF Metadata Assessment Working Group, n.d.). Each topic was assigned scores for its relations, occurrences, and the number of books the topic appears in. The score was calculated by dividing a particular topic's number of relations, occurrences, or books by the maximum for that measure in the dataset (for example, a topic's number of relations divided by the number of relations for the topic with the most relations). The overall importance score is the average of the three scores. The "topic importance score" was calculated in Tableau, which was used to visualize and sort the list of topics. From Tableau, three spreadsheets were exported:

1. Topics with link scores between 4 and $67 \%$

2. Topics with link scores between 0 and $3 \%$ and more than 10 relations

3. Topics with link scores between 0 and $3 \%$ and 2 or more books 
The three spreadsheets were combined using the command line to generate one file of 5,740 important topics. Visualizations made in Tableau are available on the Digital Production Editor's profile page of visualization workbooks (Provo, n.d.).

\section{Matching topics to external vocabularies}

Beyond connecting the monographs included in ENM with one another, one of the goals of the ENM project was to explore how to network the monographs with external resources. To experiment in this area, as Digital Production Editor I sought to match the topic map to published vocabularies, with the idea that this would eventually enable users to navigate to related information on the web (a core principle of Linked Open Data and the Semantic Web). The 5,740 important topics identified were targeted for enrichment by matching, or reconciling, to Uniform Resource Identifiers (URIs) in external controlled vocabularies such as Wikidata, the Virtual International Authority File (VIAF), Library of Congress Authorities (LC), and Faceted Application of Subject Terminology (FAST).

Using the open source software tool OpenRefine, 3,048 topics were matched with at least one URI. This represents $53 \%$ of the "important" subset and $7 \%$ of the entire topic map. Though this may not seem like a significant proportion, it should be noted that many topics would not be expected to be found in published vocabularies due to their granularity and specificity (for example, a topic such as Hurston, Zora Neale -- authorial voice -- as "mythic agency" or "occult spiritual authority").

The process of matching was labor-intensive, as it involved both checking the quality and accuracy of automated matches as well as manually selecting a match when an automated match was not possible. The URI workflow tested both coverage and performance of reconciliation software; it therefore may have skewed toward Wikidata since that vocabulary was reconciled first, and that reconciliation service was easiest to use. From our experience, it seems that automated matches of person records in Library of Congress and FAST may be more prone to error than Wikidata. This could be due to the way each reconciliation service is coded, but it could also be a reflection of the focus of LC and FAST: although LC, FAST, and Wikidata all include concepts (or subjects), LC and FAST (which derives from LC) have a dedicated name authority vocabulary included, whereas Wikidata's scope is more broad. This may mean that there are more names in LC and FAST, or a greater number of similar names and thus increased false positives. 

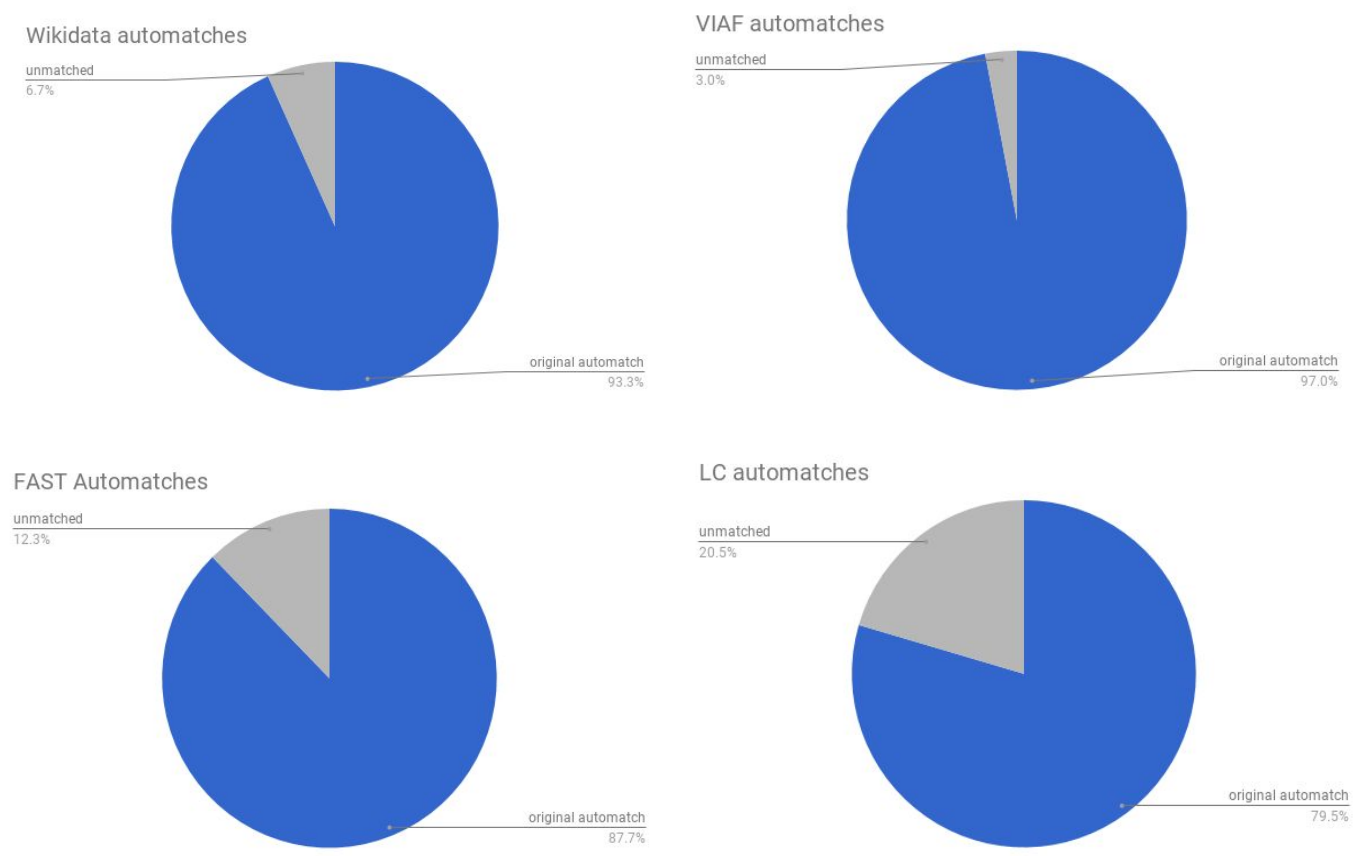

Figure 8. Charts showing unmatched automatches.

Figure 8 shows the proportion of automated matches that were unmatched in each vocabulary. It should be noted, though, that FAST and LC had a greater total of automatches than the other vocabularies, which could explain why more were unmatched.

Though further and more rigorous analysis would be required to draw conclusions, the experience of matching URIs to topics was an opportunity to reflect on the issue of whether we can leave automated matching processes alone, and whether detailed quality control of the automated matches is needed. Project principles of minimal editor involvement and looser semantics for the purposes of serendipity have to be balanced with the value of accuracy. As well, the process raised questions about the value of including URIs in just a small subset of topics; for example, is such unevenness confusing to users, or is it acceptable given that some topics are simply impossible to match with vocabulary terms because of their contextual nature and specificity? Though they do not participate in a wider web, unmatchable terms contribute their own value to the network by providing access to unique concepts and terminology that are not represented in other vocabularies.

Work on matching to URIs also fed back into more conventional editing of the topic map in TCT, in that it revealed problems with topic names, as well as topics that need to be merged or split. Thus, not only was this workflow useful for adding data such as URIs, but also for bringing focus to this subset of topics so that they could be edited according to the principles and practices outlined above. Once again, however, we came back to the question of how much messiness or imperfection is acceptable. Is it enough to just work on the subsets of topics identified? 


\section{Topics for end-users}

To begin investigating how users might react to the topic map data, the ENM project team built a prototype website using the topic map data outlined above. As noted previously, one of the principles of the project was to "pay attention to the impact of topic map editing on the user." In the spirit of this principle, we also conducted research into user expectations about experiences with ebooks. The report and literature review on Ebook user expectations produced by the Digital Production Editor drew especially on a usability evaluation of the NYU Press Open Access Books site that inspired ENM, and a white paper by JSTOR Labs on the future of digital monographs (Brown et. al., 2017).

In the Ebook expectations report, one goal was to probe how indexes either already or might in the future play into several themes of what users want to do with Ebooks. Indexes were deemed relevant to three themes identified in the report: navigating via internal links, full text search, and navigating a collection.

\section{Theme: navigating via internal links}

The EPUBs ENM presents all have hyperlinked indexes that the user can access via the project's Ebook reader, Readium. However, though there are several studies that indicate that back-of-book indexes may be better at getting users to the information they want, users do not always turn to them first or necessarily perceive them as relevant or more helpful. This is one reason the project team was interested in thinking about other ways to use the terms found in our indexes; if indexes are useful but not always the first thing on a Ebook user's mind, could we put the content in a different form that might be useful in a new way?

\section{Theme: Full text search}

Barnum et al. report that users encounter too many search results or irrelevant results because of the presence of homonyms (Barnum et al., 2004, pp. 187-188). Some studies have found that users sometimes look at the table of contents and/or index to get ideas for terms to use in a search query. Mary Coe notes in her study of book index users that they "did not always consider whether the search term they had chosen from the index would exist in that form in the text" (Coe, 2015, p. 97). The ENM team speculated that including ENM topics in the full-text search index might help address this problem. Some researchers have noted that subject headings are valuable "not as verbatim search expressions, but as sources for frequently unique keyword material" (Garrett, 2007, 72). However, one limitation of subject headings is "a general trend towards vague and general subject headings" (Duffy, 2013, p. 10). Our topics, on the other hand, are based on more specific index terms, which could address this limitation.

\section{Theme: navigating a collection}


The JSTOR Labs white paper on digital monographs highlights the importance of serendipitous discovery, or the library stacks effect. When prototyping ideas, JSTOR Labs found that their small sample of users was most interested in "The Book-as-Portal-to-Other-Scholarship" and "Topic Explorer", both of which address the experience of navigating to related content (Brown 2016, p. 13). One study by Abdullah and Gibb (2008) suggests including indexes and table of contents in library catalogues. In a paper and case study about finding primary sources in HathiTrust, Eamon Duffy (2013) remarks on how its combination of bibliographic records and full-text search can improve discovery. Besides including subject headings in search, Duffy's other suggestion is to give "the option to browse the LCSH thesaurus as one can in traditional OPACs." (Duffy, 2013, p. 11). We thought about accomplishing this through a browse topics feature in the ENM interface.

\section{ENM prototypes}

While part of ENM does involve a page-turn style Ebook reading experience, to address the goals of showcasing and experimenting with topics, we created prototypes for searching and browsing. In these prototypes, we were excited to think about activating the intellectual work of indexers in a new way. The research that went into developing the themes explained above informed the development of user stories and design objective cards, led by the ENM team's designer. The objectives the team sought to address using topics included controlling the boundaries of a search set, enabling serendipity, offering suggestions, and providing an overview of a book.
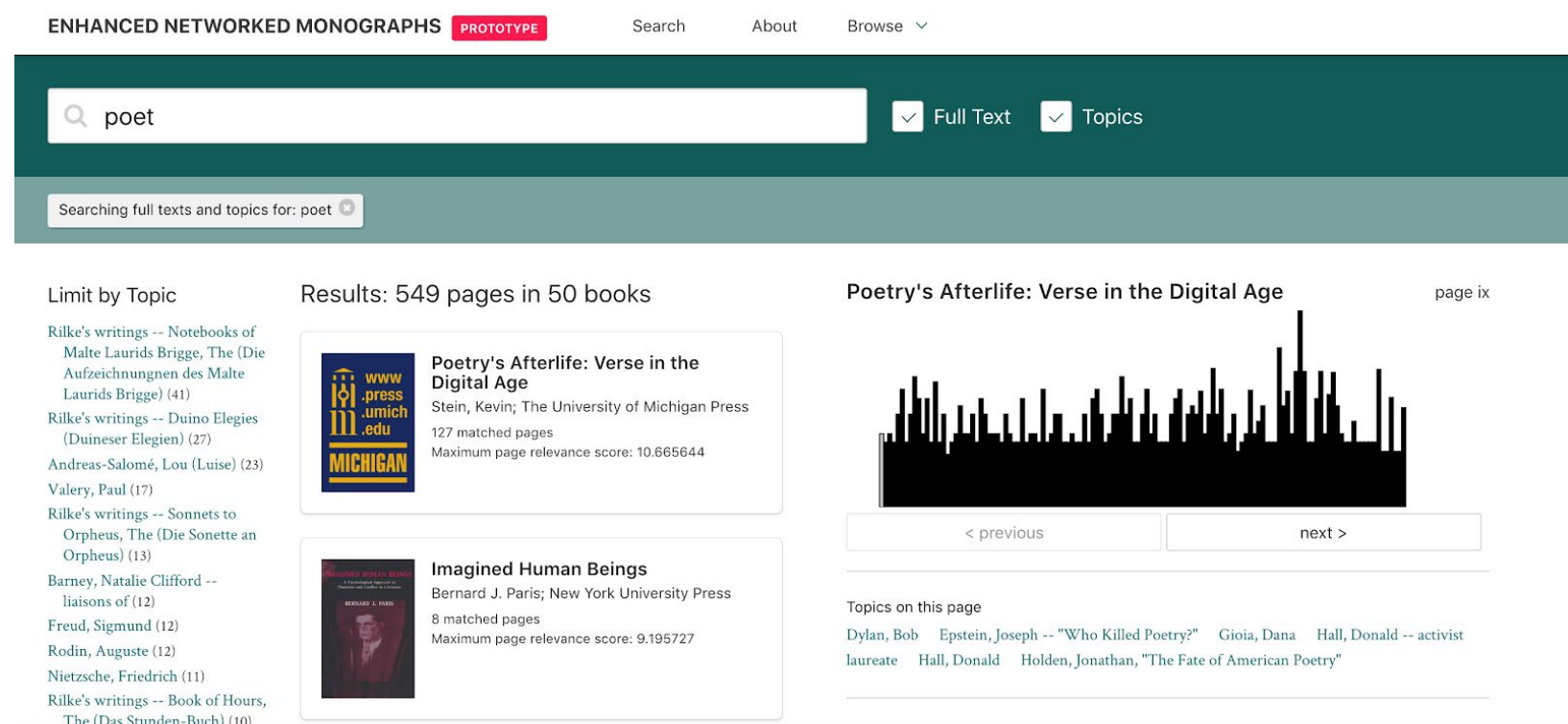

Results: 549 pages in 50 books
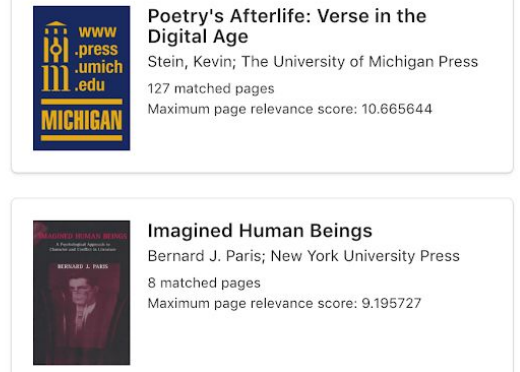

NYU PRESS $•$ NYU DLTS

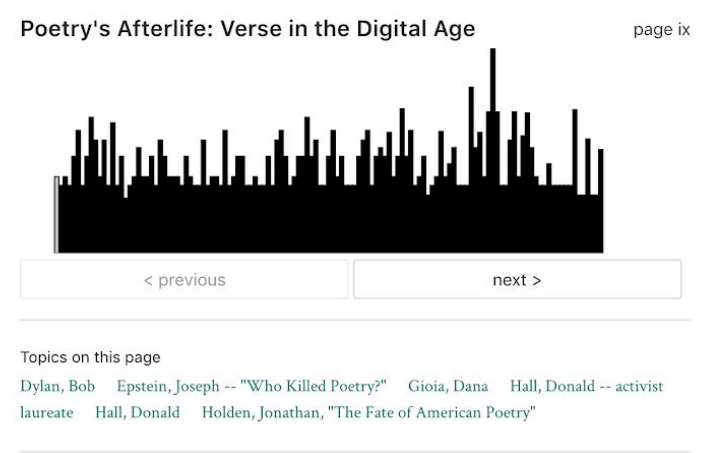

Figure 9. ENM search prototype 
The search prototype (figure 9, available via the projects section on http://dlib.nyu.edu) allows the user to enter a query that searches full text, topics, or both, and to view results grouped by book. The user can also facet by topic on the left, and view a preview of pages that match on the right. This prototype centers the book in the research experience, but tries to make use of topics to do so in order to address some of the objectives identified. For example, the filtering the user can do with facets enables them to control the boundaries of the search set; the preview gives an overview of the book and possibly serendipitous exploration.

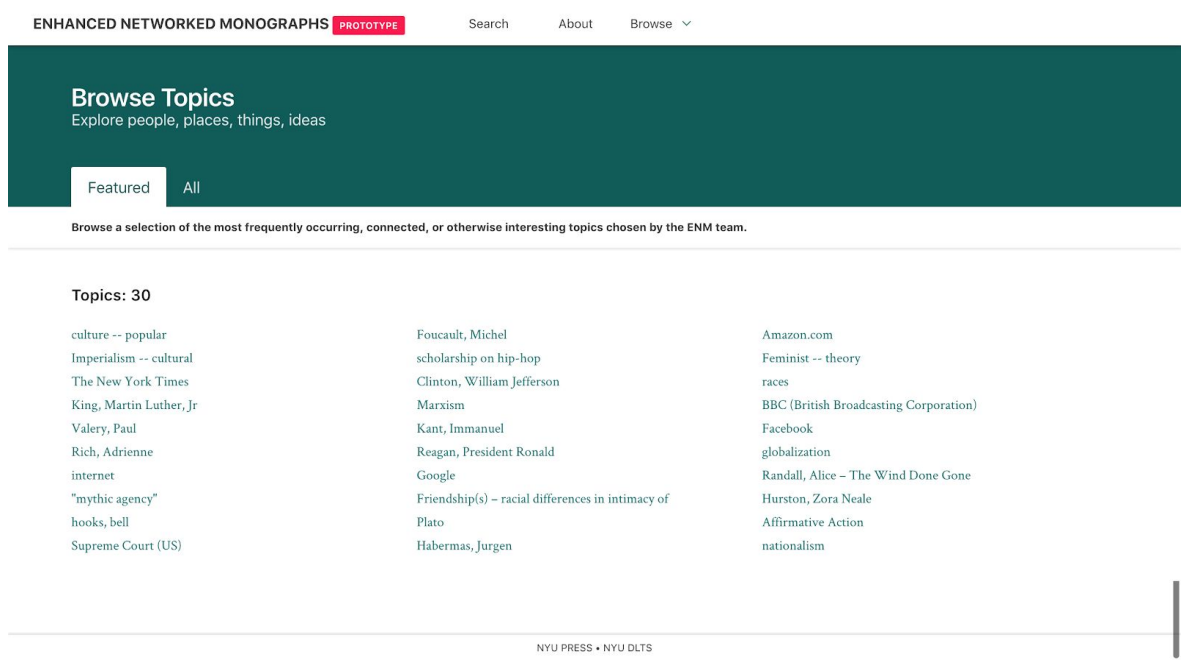

Figure 10. ENM Browse prototype showing featured topics.

Another section of the prototype website focuses on browsing (figure 10). Similar to the TCT, we provide alphabetical lists of topics and a featured topics list. This prototype puts the focus on the topics. Also like the TCT, each topic also has its own page, but with a graph visualization that allows for clicking through to related topics and links to external vocabularies (figure 11).

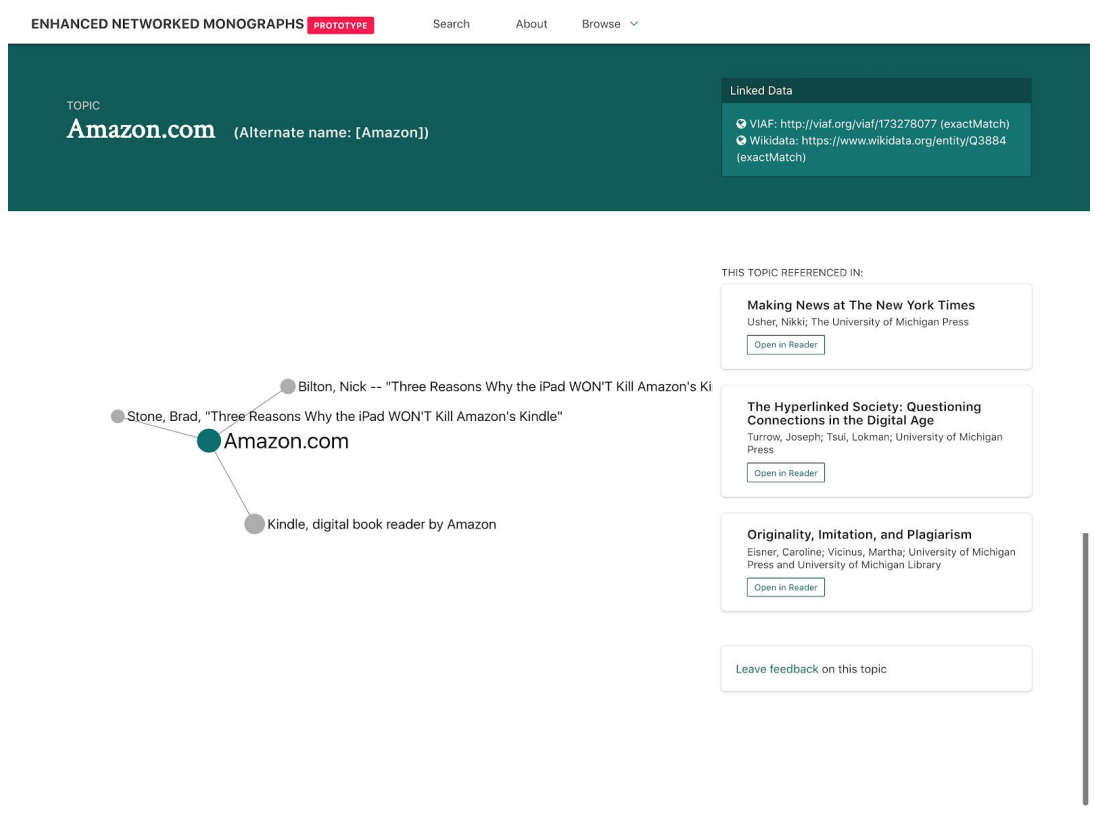

Figure 11. ENM topic page for Amazon.com showing graph visualization and external links. 


\section{Future work}

There are several opportunities for future work and improvements based on the ENM project. These recommendations and ideas derive from the experiences of working with the topic map data and designing the prototype interfaces, exploring relevant literature, and feedback from indexers at the ASI annual meeting in April 2018.

On the technical side, topic map generation and management could be made easier in some cases by improving the markup of the source EPUB index files themselves. For indexes that already exist, remediation could be done to ensure consistent use of punctuation in entries, since the TCT relies on such patterns to parse properly. For new content, the EPUB Indexes 1.0 Recommended Specification (International Digital Publishing Forum, 2015) is recommended, as it outlines distinct XML elements for each component of of the index entry (entry, locator, and cross-reference). Currently, page numbers in EPUBs are indicated in a variety of fashions, so locator data could also benefit from standardization.

For index content, modifications to convention would prepare index entries to move beyond the context of the book and participate in larger networks. For example, spelling out abbreviations, especially of person names, would help with integration and matching by reducing ambiguity. Relatedly, consistency among names within an index would be helpful; for example, in the book The Prophetic Tradition in Radical America, Eugene Debs is referred to simply as Debs in many entries, probably for economy of space. If the full name were spelled out, an automated tool like the Topic Curation Toolkit would be able to generate a link between these specific entries and the entry for Eugene Debs. Under the umbrella of spelling things out, when creating compound entries (such as advertising/advertisements), it would be helpful to use a slash instead of parentheses for multiple parts of speech in an entry, so that automated tools can recognize the entire word: for example, instead of Behavior(al), entering Behavior/behavioral.

Issues of scale arose throughout the project, and some of these could be addressed in future work. As mentioned above, the ENM corpus of texts was not domain-specific, but rather a collection of texts from several different domains of knowledge. Future projects might be more successful and less labor-intensive if they were to focus on more defined domains of knowledge, as this might mitigate homographs and other semantic issues. In the vein of Indexer's Legacy and the ASI publications mashup, indexes from a defined domain could be extracted using the TCT or similar software to create controlled vocabularies. For new content, it would be interesting to experiment with indexes created by a single indexer or indexer team. Besides providing greater consistency, such an approach could also be an opportunity to prepare index terms for machine processing from the very beginning of the project, perhaps even matching them with external controlled vocabularies from the outset. 
Computational methods such as natural language processing and topic modeling could also yield fruitful overlap with machine-actionable indexes. Topic modeling involves the use of algorithms to identify clusters of thematically related portions of texts (Blei, 2012). As noted in Blei's overview, research is being done into incorporating structured metadata into topic models, and supervised topic modeling is another technique involving pre-labelling documents. Indexes treated as metadata for entire books, or at the page level, could intersect with topic modeling in interesting ways. One recent example of such investigation is the Networked Corpus project (Binder and Jennings, n.d.), which compares the index of Adam Smith's The Wealth of Nations with a topic model trained on the text. There is also room for integrating more natural language processing techniques in the creation of relationship links between topics, for example improved stemming and lemmatization to isolate root words, which might improve confidence in automated relationship generation. Finally, future work could also further investigate the impacts of index terms. Search could be investigated by developing formal testing procedures for assessing how information retrieval is affected by the presence of indexes and index entries.

\section{References}

Abdullah, N., \& Gibb, F. (2008). Using a task-based approach in evaluating the usability of BoBls in an e-book environment. In European Conference on Information Retrieval (pp. 246-257). Springer. Retrieved from http://link.springer.com/10.1007/978-3-540-78646-7 24

Ambler, S. W. (2002). Introduction To Agile Modeling (AM). Retrieved from https://web.archive.org/web/20050512183104/http://ronin-intl.com/publications/agileModeling.pd $\underline{f}$

ASI Publications Index Mashup. (n.d.). Retrieved October 26, 2018, from https://www.asindexing.org/wp-content/uploads/2013/08/mashupa.html

Barnum, C., Henderson, E., Hood, A., \& Jordan, R. (2004). Index Versus Full-text Search: A Usability Study of User Preference and Performance. Technical Communication, 51(2), 185-206.

Binder, J., \& Jennings, C. (n.d.). Networked Corpus. Retrieved September 27, 2018, from http://networkedcorpus.com/

Blei, D. M. (2012). Probabilistic topic models. Communications of the ACM, 55(4), 77. https://doi.org/10.1145/2133806.2133826

Brown, L., Humphreys, A., Loy, M., Snyder, R., \& Spencer, C. (2017). Reimagining the Digital Monograph: Design Thinking to Build New Tools for Researchers, A JSTOR Labs Report. Retrieved from https://hcommons.org/deposits/item/hc:14411/ 
Coe, M., (2015). What do readers expect from book indexes and how do they use them? An exploratory user study. Indexer, 33(3), 90-101.

Duffy, E. P. (2013). Searching HathiTrust: Old Concepts in a New Context. Partnership: The Canadian Journal of Library \& Information Practice \& Research, 8(1), 1-13.

DLF Metadata Assessment Working Group, Environmental Scan: Publications. Retrieved from https://dlfmetadataassessment.github.io/EnvironmentalScan/entries/33publications.html

Friedman, B., Kahn, Jr., P. H., \& Borning, A. (2006). Value Sensitive Design and Information Systems. In P. Zhang \& D. F. Galletta (Eds.), Human-computer Interaction and Management Information Systems: Foundations (pp. 348-371). Armonk, UNITED STATES: Taylor and Francis. Retrieved from http://ebookcentral.proquest.com/lib/nyulibrary-ebooks/detail.action?doclD=302451

Garrett, J. (2007). Subject Headings in Full-Text Environments: The ECCO Experiment. College \& Research Libraries, 68(1), 69-81. https://doi.org/10.5860/crl.68.1.69

Huggett, Michael, and Edie Rasmussen. "Creating Meta-Indexes for Digital Domains." Proceedings of the 11th Annual International ACM/IEEE Joint Conference on Digital Libraries, ACM, 2011a, pp. 423-424. ACM Digital Library, doi:10.1145/1998076.1998166.

Huggett, Michael, and Edie Rasmussen. "The Meta-Dex Suite: Generating and Analyzing Indexes and Meta-Indexes." Proceedings of the 34th International ACM SIGIR Conference on Research and Development in Information Retrieval, ACM, 2011b, pp. 1285-1286. ACM Digital Library, doi:10.1145/2009916.2010162.

Huggett, Michael, and Edie Rasmussen. "Dynamic Online Views of Meta-Indexes." Proceedings of the 12th ACM/IEEE-CS Joint Conference on Digital Libraries, ACM, 2012, pp. 233-236. ACM Digital Library, doi:10.1145/2232817.2232860.

International Digital Publishing Forum, EPUB Indexes 1.0. (n.d.). Retrieved October 26, 2018, from http://www.idpf.org/epub/idx/

Newcomb, Steven R. "A Perspective on the Quest for Global Knowledge Interchange.” XML Topic Maps: Creating and Using Topic Maps for the Web, edited by Jack Park and Sam Hunting, Addison-Wesley, 2003, pp.37-38.

Ochoa, Xavier, and Erik Duval. "Automatic Evaluation of Metadata Quality in Digital Repositories." International Journal on Digital Libraries, vol. 10, no. 2-3, Aug. 2009, p. 67. link.springer.com, doi:10.1007/s00799-009-0054-4. 
Provo, Alexandra. Alex - Profile | Tableau Public. (n.d.). Retrieved October 26, 2018, from https://public.tableau.com/profile/alex2378\#!/

Ream, D. K., \& Wyman, P. (2014). ASI Publications Revamp: eBooks and an Index Mashup, 22(11), 2. 